\title{
ON THE 2-NILPOTENT MULTIPLIER OF FINITE $p$-GROUPS
}

\author{
PEYMAN NIROOMAND \\ School of Mathematics and Computer Science, \\ Damghan University, Damghan 36715-364, Iran \\ e-mail:niroomand@du.ac.ir,p_niroomand@yahoo.com \\ and MOHSEN PARVIZI \\ Department of Pure Mathematics, Ferdowsi University of Mashhad, \\ Mashhad 91775, Iran \\ e-mail:parvizi@math.um.ac.ir
}

(Received 15 October 2012; revised 29 May 2014; accepted 11 August 2014)

\begin{abstract}
The purpose of this paper is a further investigation on the 2-nilpotent multiplier, $\mathcal{M}^{(2)}(G)$, when $G$ is a non-abelian $p$-group. Furthermore, taking $G$ in the class of extra-special $p$-groups, we will get the explicit structure of $\mathcal{M}^{(2)}(G)$ and will classify 2-capable groups in that class.
\end{abstract}

2010 Mathematics Subject Classification. 20C25, 20 D15.

1. Motivation. Let $G$ be a finite group presented as the quotient of a free group $F$ by a normal subgroup $R$. Then the 2-nilpotent multiplier of $G$ is defined as the abelian group

$$
\mathcal{M}^{(2)}(G)=\frac{R \cap \gamma_{3}(F)}{[R, F, F]}
$$

which is a lesser extent of the Baer invariant of group $G$ with respect to the variety of nilpotent groups of class at most $c, \mathcal{M}^{(c)}(G)$, and it has been introduced in [1] (see also $[8,10,18]$ for more details). In the case $c=1$, the Baer invariant of $G$ is denoted by $\mathcal{M}(G)$ and it is called the Schur multiplier of $G$. There are wide stories involving this concept and it can be found for instance in $[2,9,10,13,14,15,22,23,24]$.

Information about the 2-nilpotent multiplier of $G$ may be used as an instrument in connection to isologism of groups (see [6]). Recall that the concept of isologism of groups was introduced by $\mathrm{P}$. Hall in $[\mathbf{1 1}, \mathbf{1 2}]$ and it is used for classifying finite $p$-groups into isologism classes.

Thanks to [6, Theorem 3.1], we can easily obtain the order of $\mathcal{M}^{(2)}(G)$ when $G$ is a finite abelian group. Unfortunately, in the case of non-abelian groups, there is no explicit instrument to compute the structure of $\mathcal{M}^{(2)}(G)$ for an arbitrary non-abelian group $G$. In the way of computation in more classes of groups, here we obtain the explicit structure of 2-nilpotent multipliers of all extra-special $p$-groups. In the other direction, it seems finding a suitable upper bound may be useful to know more about the 2-nilpotent multiplier and 2-capability of groups. 
It is shown in $[\mathbf{1 9}, \mathbf{2 0}]$ that for a $p$-group $G$ of order $p^{n}$,

$$
\left|\mathcal{M}^{(2)}(G)\right|\left|\gamma_{3}(G)\right| \leq\left|\mathcal{M}^{(2)}\left(\mathbb{Z}_{p}^{(n)}\right)\right|
$$

It jointly with Theorem 3.2 shows $\left|\mathcal{M}^{(2)}(G)\right|$ is bounded by $p^{\frac{1}{3} n(n-1)(n+1)}$. We will show that the bound is attained exactly when $G$ is elementary abelian similar to the result of [2, Corollary 2] due to Berkovich. Although we will reduce the upper bound as much as possible in the case of non-abelian $p$-groups, and as a result we will generalize the work of Berkovich [2] to the 2-nilpotent multipliers of finite $p$ groups.

In this paper, we characterize the explicit structure of the 2-nilpotent multiplier of all extra-special $p$-groups as same as [15, Theorem 3.3.6] for the Schur multiplier by a quite different technique, and then we will find a suitable upper bound for the order of $\mathcal{M}^{(2)}(G)$. Moreover, we will state necessary and sufficient conditions for a group to obtain the given upper bound. Finally, we extend the result of [3] about the capability of extra-special $p$-groups to 2-capability.

2. Preliminaries. This section deals with prerequisite concepts and results, which will be used in the next section.

We use techniques involving the concept of basic commutators. Here is the definition.

Let $X$ be an arbitrary subset of a free group, and select an arbitrary total order for $X$. The basic commutators on $X$, their weight $w t$, and the ordering among them are defined as follows:

(i) The elements of $X$ are basic commutators of weight one, ordered according to the total order previously chosen.

(ii) Having defined the basic commutators of weight less than $n$, a basic commutator of weight $n$ is $c=[b, a]$, where:

(a) $b$ and $a$ are basic commutators and $w t(b)+w t(a)=n$, and

(b) $b>a$, and if $b=\left[b_{1}, b_{2}\right]$, then $a \geq b_{2}$.

(iii) The basic commutators of weight $n$ follow those of weight less than $n$. The basic commutators of weight $n$ are ordered among themselves in any total order, but the most common used total order is lexicographic order, that is if $\left[b_{1}, a_{1}\right]$ and $\left[b_{2}, a_{2}\right]$ are basic commutators of weight $n$, then $\left[b_{1}, a_{1}\right]<\left[b_{2}, a_{2}\right]$ if and only if $b_{1}<b_{2}$ or $b_{1}=b_{2}$ and $a_{1}<a_{2}$.

There are some classical results involving $\mathcal{M}^{(2)}(G)$ as follows.

Theorem 2.1. Let $G$ be a finite group and $B \unlhd G$. Set $A=G / B$.

(i) [7, Proposition 2] If $B \subseteq Z_{2}(G)$, then
(a) $\left|\mathcal{M}^{(2)}(G)\right|\left|B \cap \gamma_{3}(G)\right|$ divides $\left|\mathcal{M}^{(2)}(A)\right|\left|\left(B \otimes \frac{G}{\gamma_{3}(G)}\right) \otimes \frac{G}{\gamma_{3}(G)}\right|$,
(b) [16] The sequence
$(B \wedge G) \wedge G \rightarrow \mathcal{M}^{(2)}(G) \rightarrow \mathcal{M}^{(2)}(G / B) \rightarrow B \cap \gamma_{3}(G) \rightarrow 1$ is exact.

(ii) $[19]\left|\mathcal{M}^{(2)}(A)\right|$ divides $\left|\mathcal{M}^{(2)}(G)\right|\left|B \cap \gamma_{3}(G)\right| /|[[B, G], G]|$.

The following theorem plays an essential role in the rest. 
THEOREM 2.2 [21]. Let $G$ and $H$ be finite groups. Then there is a natural isomorphism

$$
\mathcal{M}^{(2)}(G \times H) \cong \mathcal{M}^{(2)}(G) \times \mathcal{M}^{(2)}(H) \times\left(G^{a b} \otimes G^{a b}\right) \otimes H^{a b} \times\left(H^{a b} \otimes H^{a b}\right) \otimes G^{a b} .
$$

In which $X^{a b}=X / X^{\prime}$ for a group $X$.

We use the following theorem several times in the paper and it can be found in [17, Theorem 2.4] for $c$-nilpotent multiplier, but in abelian $p$-groups there is a simpler proof, which is also in [6, Theorem 3.1].

THEOREM 2.3. Let $G=\mathbb{Z}_{m^{m_{1}}} \oplus \mathbb{Z}_{p^{m_{2}}} \oplus \cdots \oplus \mathbb{Z}_{p} m_{k}$, where $m_{1} \geq m_{2} \geq \cdots \geq m_{k}$. Then

$$
\mathcal{M}^{(2)}(G) \cong \bigoplus_{i=2}^{k} \mathbb{Z}_{p^{m_{i}}}^{\left(i^{2}-i\right)} .
$$

Proof. We proceed by induction on $k$. For $k=2$, Theorem 2.2 implies that

$$
\begin{aligned}
\mathcal{M}^{(2)}\left(\mathbb{Z}_{p^{m_{1}}} \oplus \mathbb{Z}_{p^{m_{2}}}\right) \cong \mathcal{M}^{(2)}\left(\mathbb{Z}_{p^{m_{1}}}\right) \oplus \mathcal{M}^{(2)}\left(\mathbb{Z}_{p^{m_{2}}}\right) \oplus\left(\left(\mathbb{Z}_{p^{m_{1}}} \otimes \mathbb{Z}_{p^{m_{1}}}\right) \otimes \mathbb{Z}_{p^{m_{2}}}\right) \\
\oplus\left(\left(\mathbb{Z}_{p^{m_{2}}} \otimes \mathbb{Z}_{p^{m_{2}}}\right) \otimes \mathbb{Z}_{p^{m_{1}}}\right) \cong \mathbb{Z}_{p^{m_{2}}}^{(2)} .
\end{aligned}
$$

Assume that $G=\mathbb{Z}_{p^{m_{1}}} \oplus \mathbb{Z}_{p^{m_{2}}} \oplus \cdots \oplus \mathbb{Z}_{p^{m_{k}}} \oplus \mathbb{Z}_{p^{m_{k+1}}}$ and put $G_{1}=\mathbb{Z}_{p^{m_{1}}} \oplus \mathbb{Z}_{p^{m_{2}}} \oplus$ $\cdots \oplus \mathbb{Z}_{p}{ }_{p}$. Since $G=G_{1} \oplus \mathbb{Z}_{p}{ }^{m_{k+1}}$, Theorem 2.2 deduces that

$$
\mathcal{M}^{(2)}(G) \cong \mathcal{M}^{(2)}\left(G_{1}\right) \oplus\left(\left(G_{1} \otimes G_{1}\right) \otimes \mathbb{Z}_{p}^{m_{k+1}}\right) \oplus\left(\left(\mathbb{Z}_{p}{ }_{m_{k+1}} \otimes \mathbb{Z}_{p}{ }^{m_{k+1}}\right) \otimes G_{1}\right),
$$

which is isomorphic to

$$
\bigoplus_{i=2}^{k} \mathbb{Z}_{p^{m_{i}}}^{\left(i^{2}-i\right)} \oplus \mathbb{Z}_{p^{m_{k+1}}}^{\left(k^{2}\right)} \oplus \mathbb{Z}_{p^{m_{k+1}}}^{(k)}=\bigoplus_{i=2}^{k+1} \mathbb{Z}_{p^{m_{i}}}^{\left(i^{2}-i\right)} .
$$

The result holds.

Throughout this paper, $G^{(k)}$ is using to denote the direct product of $k$-copies of the group $G$.

3. 2-Nilpotent multipliers of finite $p$-groups. This section is devoted to $\mathcal{M}^{(2)}(G)$, when $G$ is a finite $p$-group. It was proved in $[\mathbf{1 9}, \mathbf{2 0}]$ that the order of the 2-nilpotent multiplier of a $p$-group $G$ of order $p^{n}$, is at most $\left|\mathcal{M}^{(2)}\left(\mathbb{Z}_{p}^{(n)}\right)\right| /\left|\gamma_{3}(G)\right|$, now Theorem 3 implies $\left|\mathcal{M}^{(2)}(G)\right|$ to be bounded by $p^{\frac{1}{3} n(n-1)(n+1)}$. Looking the upper bound and the result, which will be given in the next (see Theorem 3.7), we can see that the elementary abelian groups attain the upper bound as same as the result of Berkovich in [2] for the Schur multiplier of $p$-groups. Roughly speaking, we are interested to reduce this bound as much as possible when $G$ is a non-abelian $p$-group. Our content extends the result of [22] for the Schur multiplier of finite $p$-groups.

Let $D_{8}$ and $Q_{8}, E_{1}$ and $E_{2}$ denote the dihedral and quaternion groups of order 8 and the extra-specials of order $p^{3}$ and exponent $p$ and $p^{2}$, respectively. 
We need the concept of the exterior square of a group. Here a brief description is presented. The exterior square $G \wedge G$ is a factor group of the tensor square of a group $G$. The tensor square is a special case of non-abelian tensor product of two groups when the two groups coincide and the action is conjugation. More precisely, it is generated by the symbols $g \otimes h$ subject to the relations

$$
g g^{\prime} \otimes h=\left({ }^{g} g^{\prime} \otimes{ }^{g} h\right)(g \otimes h) \text { and } g \otimes h h^{\prime}=(g \otimes h)\left({ }^{h} g \otimes{ }^{h} h^{\prime}\right)
$$

for all $g, g^{\prime}, h, h^{\prime} \in G$, where ${ }^{g} g^{\prime}=g g^{\prime} g^{-1}$. The exterior square $G \wedge G$ is obtained by imposing the additional relation $g \otimes g=1_{\otimes}$ on $G \otimes G$. The image of $g \otimes h$ in $G \wedge G$ is denoted by $g \wedge h$ for all $g, h \in G$ (see [4] Section 2 and [5] p. 181 for more details). Recall that the exterior centre $Z^{\wedge}(G)$ of $G$ is equal to the set of all elements $g \in G$ such that $g \wedge h=1_{G \wedge G}$ for all $h \in G$. Already [8, Proposition 16 (i)] shows that $Z^{\wedge}(G)$ is a central subgroup of $G$, which allows us to decide whether $G$ is a capable group, that is whether $G$ is isomorphic to $E / Z(E)$ for some group $E$. In fact $G$ is capable if and only if $Z^{\wedge}(G)=1$ (see [8] ). It is known by [3] that the only capable extra-special $p$-groups are $E_{1}$ and $D_{8}$.

The following Theorem determines the explicit structure of the Schur multipliers of all extra-special $p$-groups. We will state and prove a similar one for the 2-nilpotent multipliers of all extra-special $p$-groups in the rest of the paper.

TheOREM 3.1 Beyl and Tappe 1982 [15, Theorem 3.3.6]. Let $G$ be an extra-special p-group of order $p^{2 n+1}$.

(i) If $n>1$, then $G$ is unicentral and $\mathcal{M}(G)$ is an elementary abelian p-group of order $p^{2 n^{2}-n-1}$.

(ii) Suppose that $|G|=p^{3}$ and $p$ is odd. Then $\mathcal{M}(G)=\mathbb{Z}_{p} \times \mathbb{Z}_{p}$ if $G$ is of exponent $p$ and $\mathcal{M}(G)=0$ if $G$ is of exponent $p^{2}$.

(iii) The quaternion group of order 8 has trivial multiplier, whereas the multiplier of the dihedral group of order 8 is of order 2.

First of all the 2-nilpotent multipliers of all non-capable extra-special $p$-groups can be computed thanks to Theorem 2.1 (b).

THEOREM 3.2. Let $G$ be a non-capable extra-special p-group of order $p^{n}$. Then

$$
\mathcal{M}^{(2)}(G) \cong \mathcal{M}^{(2)}\left(G / G^{\prime}\right)
$$

and is isomorphic to $\mathbb{Z}_{p}^{\left(\frac{1}{3}\left(8 n^{3}-2 n\right)\right)}$.

Proof. Since $G$ is a non-capable extra-special $p$-group, $Z^{\wedge}(G)=G^{\prime}$. Now by virtue of Theorem 2.1(b), the sequence

$$
\left(G^{\prime} \wedge G\right) \wedge G \stackrel{\iota}{\rightarrow} \mathcal{M}^{(2)}(G) \rightarrow \mathcal{M}^{(2)}\left(G / G^{\prime}\right) \rightarrow G^{\prime} \cap \gamma_{3}(G) \rightarrow 1
$$

is exact. Since $G$ is nilpotent of class 2 and $\operatorname{Im} \iota=0$, we have $\mathcal{M}^{(2)}(G) \cong \mathcal{M}^{(2)}\left(G / G^{\prime}\right)$. But $G / G^{\prime}$ is elementary abelian of rank $2 n$, so Theorem completes the proof.

The only extra-special $p$-groups, which the 2-nilpotent multipliers needs to be computed are $E_{1}$ and $D_{8}$. In the following we intend to compute $\mathcal{M}^{(2)}\left(E_{1}\right)$, where $E_{1}$ is the extra-special $p$-group of order $p^{3}$ and exponent $p$ with the presentation

$$
E_{1}=\left\langle x, y \mid x^{p}=y^{p}=[y, x]^{p}=[y, x, y]=[y, x, x]=1\right\rangle .
$$


We have $\mathcal{M}^{(2)}(G)=\frac{R \cap \gamma_{3}(F)}{[R, F, F]}$ in which $F$ is the free group on the set $\{x, y\}$ and $R=$ $\left\langle x^{p}, y^{p},[y, x]^{p},[y, x, y],[y, x, x]\right\rangle^{F}$. Since $E_{1}$ is nilpotent of class 2 , we have $\gamma_{3}(F) \subseteq R$ and hence

$$
\mathcal{M}^{(2)}(G)=\frac{R \cap \gamma_{3}(F)}{[R, F, F]} \cong \frac{\gamma_{3}(F) / \gamma_{5}(F)}{[R, F, F] / \gamma_{5}(F)}
$$

We know that $\gamma_{3}(F) / \gamma_{5}(F)$ is the free abelian group with the basis of all basic commutators of weights 3 and 4 on $\{x, y\}$, that is the set

$$
\{[y, x, x],[y, x, y],[y, x, x, x],[y, x, x, y],[y, x, y, y]\} \text { in which } y>x .
$$

We will find a suitable generating set for $[R, F, F] / \gamma_{5}(F)$ to show that

$$
\mathcal{M}^{(2)}\left(E_{1}\right) \cong \mathbb{Z}_{p}^{(5)}
$$

LEMMA 3.3. With the above notations and assumptions the following congruences hold modulo $\gamma_{5}(F)(a, b, c, d \in F, n \in \mathbb{N})$.

(i) $\left[a^{n}, b, c, d\right] \equiv[a, b, c, d]^{n}$,

(ii) $\left[a^{n}, b, c\right] \equiv[a, b, c]^{n}[a, b, a, c]^{\left(\frac{1}{2}\right)}$,

(iii) $\left[[a, b]^{n}, c, d\right] \equiv[a, b, c, d]^{n}$.

\section{Proof.}

(i) we have $\left[a^{n+1}, b\right]=\left[a^{n}, b\right]\left[a^{n}, b, a\right][a, b]$ therefore

$\left[a^{n+1}, b, c\right]=\left[a^{n}, b, c\right]^{\left[a^{n}, b, a\right][a, b]}\left[a^{n}, b, a, c\right]^{[a, b]}[a, b, c]$, which is congruent to $\left[a^{n}, b, c\right]\left[a^{n}, b, a, c\right][a, b, c]$ working modulo $\gamma_{5}(F)$. Commuting the last one with $d$ gives $\left[a^{n+1}, b, c, d\right] \equiv\left[a^{n}, b, c, d\right]^{\left[a^{n}, b, a, c\right][a, b, c]}\left[a^{n}, b, a, c, d\right]^{[a, b, c]}[a, b, c, d]$ again is congruent to $\left[a^{n}, b, c, d\right][a, b, c, d]$ modulo $\gamma_{5}(F)$. Induction completes the proof.

(ii) Using the above we have $\left[a^{n+1}, b, c\right] \equiv\left[a^{n}, b, c\right]\left[a^{n}, b, a, c\right][a, b, c]\left(\bmod \gamma_{5}(F)\right)$, but $\left[a^{n}, b, a, c\right] \equiv[a, b, a, c]^{n}\left(\bmod \gamma_{5}(F)\right)$, and so

$$
\begin{aligned}
{\left[a^{n+1}, b, c\right] } & \equiv\left[a^{n}, b, c\right][a, b, a, c]^{n}[a, b, c] \\
& \equiv\left[a^{n}, b, c\right][a, b, c][a, b, a, c]^{n} \quad\left(\bmod \gamma_{5}(F)\right) .
\end{aligned}
$$

Now induction shows the result.

(iii) It is a consequence of part (ii).

We are in a position to prove the next theorem, which is a key to compute the 2-nilpotent multiplier of $E_{1}$.

THEOREM 3.4. With the above notations and assumptions we have

$[R, F, F] \equiv\left\langle[y, x, x]^{p},[y, x, y]^{p},[y, x, x, x]^{p},[y, x, x, y]^{p},[y, x, y, y]^{p}\right\rangle \quad\left(\bmod \gamma_{5}(F)\right)$.

Proof. Since $R=\left\langle x^{p}, y^{p},[y, x]^{p},[y, x, y],[y, x, x]\right\rangle^{F}$, we have

$[R, F, F]=\left\langle\left[x^{p}, a, b\right],\left[y^{p}, c, d\right],\left[[y, x]^{p}, e, f\right] \mid a, b, c, d, e, f \in F\right\rangle^{F} \quad\left(\bmod \gamma_{5}(F)\right)$, 
which is equal to

$$
\begin{aligned}
& \left\langle\left[x^{p}, a, b\right]\left[x^{p}, a, b, c\right],\left[y^{p}, d, e\right]\left[y^{p}, d, e, f\right],\left[[y, x]^{p}, g, h\right]\right. \\
& a, b, c, d, e, f, g, h \in F\rangle \quad\left(\bmod \gamma_{5}(F)\right) .
\end{aligned}
$$

But Lemma 3.3 implies that

$$
[R, F, F]=\left\langle\left[x^{p}, a, b\right],\left[y^{p}, c, d\right],\left[[y, x]^{p}, e, f\right] \mid a, b, c, d, e, f \in F\right\rangle \quad\left(\bmod \gamma_{5}(F)\right) .
$$

Using the fact that each element of $F$ can be written as the form $y^{\alpha} x^{\beta}[y, x]^{\gamma}$ modulo $\gamma_{3}(F)$, Lemma 3.3 and some commutator computations show that

$$
\begin{gathered}
{[R, F, F]=\left\langle[x, a, b]^{p}[x, a, x, b]^{\left(\frac{p}{2}\right)},[y, c, d]^{p}[y, c, y, d]^{\left(\begin{array}{c}
p \\
2
\end{array}\right)},\right.} \\
{[y, x, x, x]^{p},[y, x, x, y]^{p},[y, x, y, y]^{p}|a, b, c, d \in F\rangle \quad\left(\bmod \gamma_{5}(F)\right) .}
\end{gathered}
$$

Therefore $[y, x, y]^{p}[y, x, y, y]^{\left(\frac{p}{2}\right)} \in[R, F, F]\left(\bmod \gamma_{5}(F)\right)$ and because $p$ is an odd prime $[y, x, y]^{p} \in[R, F, F]\left(\bmod \gamma_{5}(F)\right)$. A simple use of Hall-Witt identity shows $[y, x, x]^{p} \in$ $[R, F, F]\left(\bmod \gamma_{5}(F)\right)$, as required.

All necessary information is gathered and we are ready to state and prove the following theorem.

THEOREM 3.5. With the above notations and assumptions, we have

$$
\mathcal{M}^{(2)}\left(E_{1}\right) \cong \mathbb{Z}_{p}^{(5)}
$$

Proof. The set $\{[y, x, x],[y, x, y],[y, x, x, x],[y, x, x, y],[y, x, y, y]\}$ is a basis for the free abelian group $\gamma_{3}(F) / \gamma_{5}(F)$ and $[R, F, F] / \gamma_{5}(F)$ is generated by

$$
\left\{[y, x, x]^{p},[y, x, y]^{p},[y, x, x, x]^{p},[y, x, x, y]^{p},[y, x, y, y]^{p}\right\},
$$

so the result holds.

Since $\mathcal{M}^{(2)}\left(D_{8}\right) \cong \mathbb{Z}_{2} \oplus \mathbb{Z}_{4}$ by $[\mathbf{6}]$ and the fact that $E_{2}$ and $Q_{8}$ are not capable group, we summarize explicit structure of the 2-nilpotent multipliers of all extra-special $p$ groups as follows. The following theorem is a version of Theorem 3.1 for the variety of nilpotent groups of class at most 2, note that as in Theorem 3.1 the case $|G|=p^{3}$ differs from other cases in results even in case of odd $p$.

THEOREM 3.6. Let $G$ be an extra-special p-group of order $p^{2 n+1}$.

(i) If $n>1$, then $\mathcal{M}^{(2)}(G)$ is an elementary abelian p-group of order $p^{\frac{1}{3}\left(8 n^{3}-2 n\right)}$.

(ii) Suppose that $|G|=p^{3}$ and $p$ is odd. Then $\mathcal{M}^{(2)}(G)=\mathbb{Z}_{p}^{(5)}$ if $G$ is of exponent $p$ and $\mathcal{M}^{(2)}(G)=\mathbb{Z}_{p} \times \mathbb{Z}_{p}$ if $G$ is of exponent $p^{2}$.

(iii) The quaternion group of order 8 has Klein four-group as 2-nilpotent multiplier, whereas the 2-nilpotent multiplier of the dihedral group of order 8 is $\mathbb{Z}_{2} \oplus \mathbb{Z}_{4}$.

In order to consider non-abelian $p$-groups, we need to know the behaviour of the order of $\mathcal{M}^{(2)}(G)$ for abelian $G$.

THEOREM 3.7. Let $G=\mathbb{Z}_{p^{m_{1}}} \oplus \mathbb{Z}_{p} m_{2} \oplus \cdots \oplus \mathbb{Z}_{p} m_{k}$, where $m_{1} \geq m_{2} \geq \cdots \geq m_{k}$. Then 
(i) $\left|\mathcal{M}^{(2)}(G)\right|=p^{\frac{1}{3} n(n-1)(n+1)}$ if and only if $m_{i}=1$ for all $i$.

(ii) $\left|\mathcal{M}^{(2)}(G)\right| \leq p^{\frac{1}{3} n(n-1)(n-2)}$ if and only if $m_{1} \geq 2$.

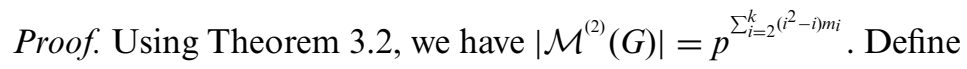

$$
S\left(k ; m_{1}, \ldots, m_{k}\right)=\sum_{i=2}^{k}\left(i^{2}-i\right) m_{i} .
$$

It is easy to see that

$$
\frac{1}{3} n(n-1)(n+1)=S(n ; 1, \ldots, 1) \text { and } \frac{1}{3} n(n-1)(n-2)=S(n-1 ; 2,1, \ldots, 1) .
$$

Assume that for some $j$, we have $m_{j}>1$ a straightforward computation shows that

$$
S\left(k ; m_{1}, \ldots, m_{k}\right)-S\left(k+1 ; m_{1}, \ldots, m_{j}-1, \ldots, m_{k}, 1\right)=(k+j)(j-k-1),
$$

which is negative. Hence the maximum value of $S\left(k ; m_{1}, \ldots, m_{k}\right)$ is $S(n ; 1, \ldots, 1)$, and the next largest value of $S\left(k ; m_{1}, \ldots, m_{k}\right)$ is $S(n-1 ; 2,1, \ldots, 1)$, which proves the theorem.

In the following we want to consider the influence of the derived subgroup on the second nilpotent multiplier, then we give an upper bound for the order of the 2-nilpotent multiplier of a finite $p$-group in terms of the order of its derived subgroup.

LEMMA 3.8. Let $G$ be a non-abelian finite p-group of order $p^{n}$ whose derived subgroup is of order $p$. Then $\left|\mathcal{M}^{(2)}(G)\right| \leq p^{\frac{1}{3} n(n-1)(n-2)+3}$, and the equality holds if and only if $G \cong$ $E_{1} \times \mathbb{Z}_{p}^{(n-3)}$.

Proof. If $G / G$ is not elementary abelian, then Theorem 2.1(a) implies that

$$
\left|\mathcal{M}^{(2)}(G)\right| \leq\left|\mathcal{M}^{(2)}\left(G^{a b}\right)\right|\left|\left(G^{\prime} \otimes G^{a b}\right) \otimes G^{a b}\right| .
$$

On the other hand, thanks to Theorem 3.7(b),

$$
\left|\mathcal{M}^{(2)}\left(G^{a b}\right)\right| \leq p^{\frac{1}{3}(n-1)(n-2)(n-3)} .
$$

Therefore $\left|\mathcal{M}^{(2)}(G)\right| \leq p^{\frac{1}{3} n(n-1)(n-2)+2}$.

Now assume that $G / G^{\prime}$ is elementary abelian. It is shown that [22, Lemma 2.2], $G$ is a central product of an extra-special $p$-group $H$ of order $p^{2 k+1}$ and $Z(G)$ of order $p^{n-2 k}$. We may assume that $|Z(G)| \geq p^{2}$ due to Theorem 3.6. If $Z(G)$ is not elementary abelian, then by Theorem 2.1(ii) we have

$$
\begin{aligned}
\left|\mathcal{M}^{(2)}(G)\right| & \leq p\left|\mathcal{M}^{(2)}(H \times Z(G))\right|=p\left|\mathcal{M}^{(2)}(H)\right| \times\left|\mathcal{M}^{(2)}(Z(G))\right| \\
& \times\left|\left(H^{a b} \otimes H^{a b}\right) \otimes Z(G)\right| \times\left|(Z(G) \otimes Z(G)) \otimes H^{a b}\right| .
\end{aligned}
$$

On the other hand, Theorems 3.2, 3.6 and 3.7 show that

$$
\left|\mathcal{M}^{(2)}(H)\right|=\left|\mathcal{M}^{(2)}\left(H / H^{\prime}\right)\right|= \begin{cases}p^{\frac{2}{3} k(2 k+1)(2 k-1)} & \text { if } k>1 \\ p^{5} & \text { if } k=1\end{cases}
$$


Since $Z(G)$ is not elementary abelian, we have

$$
\begin{gathered}
\left|M^{(2)}(Z(G))\right| \leq p^{\frac{1}{3}(n-2 k)(n-2 k-1)(n-2 k-2)}, \\
\left|\left(H^{a b} \otimes H^{a b}\right) \otimes Z(G)\right| \leq p^{4 k^{2}(n-2 k-1)} \text { and }\left|H^{a b} \otimes(Z(G) \otimes Z(G))\right| \leq p^{2 k(n-2 k-1)^{2}} .
\end{gathered}
$$

Therefore $\left|\mathcal{M}^{(2)}(G)\right| \leq p^{\frac{1}{3} 2 k(2 k+1)(2 k-1)+\frac{1}{3}(n-2 k)(n-2 k-1)(n-2 k-2)+4 k^{2}(n-2 k-1)+1}$. Now a straightforward computation shows $\left|\mathcal{M}^{(2)}(G)\right|<p^{\frac{1}{3} n(n-1)(n-2)+3}$.

Still in this case when $Z(G)$ is elementary abelian, suppose that $K$ be a complement of $G^{\prime}$ in $Z(G)$, so $Z(G)=G^{\prime} \times K$, and hence $G=H \times K$. Theorem 3.7 implies that $\mathcal{M}^{(2)}(G)=\mathcal{M}^{(2)}(H \times K)=\mathcal{M}^{(2)}(H) \times \mathcal{M}^{(2)}(K) \times(K \otimes K) \otimes H^{a b} \times\left(H^{a b} \otimes H^{a b}\right) \otimes K$.

Similar to the previous case we have

$$
\begin{gathered}
\left|\mathcal{M}^{(2)}(K)\right|=p^{\frac{1}{3}(n-2 k-1)(n-2 k-2)(n-2 k)},\left|(K \otimes K) \otimes H^{a b}\right|=p^{2 k(n-2 k-1)^{2}} \text { and } \\
\left|\left(H^{a b} \otimes H^{a b}\right) \otimes K\right|=p^{4 k^{2}(n-2 k-1)} .
\end{gathered}
$$

Some computations imply that

$$
\left|\mathcal{M}^{(2)}(G)\right|= \begin{cases}p^{\frac{1}{3} n(n-1)(n-2)} & \text { if } k>1 \\ p^{\frac{1}{3} n(n-1)(n-2)+3} & \text { if } k=1\end{cases}
$$

Now it is easy to see that $\left|\mathcal{M}^{(2)}(G)\right|=p^{\frac{1}{3 n(n-1)(n-2)+3}}$ if and only if $k=1$ and $G \cong$ $E_{1} \times \mathbb{Z}_{p}^{(n-3)}$.

Using Lemma 3.8 and induction we can prove the following Theorem.

THEOREM 3.9. Let $G$ be a p-group of order $p^{n}$ with $\left|G^{\prime}\right|=p^{m}(m \geq 1)$. Then

$$
\left|\mathcal{M}^{(2)}(G)\right| \leq p^{\frac{1}{3}(n-m)((n+2 m-2)(n-m-1)+3(m-1))+3} .
$$

In particular, we have $\left|\mathcal{M}^{(2)}(G)\right| \leq p^{\frac{1}{3} n^{(n-1)(n-2)+3}}$ and the equality holds in last one if and only if $G \cong E_{1} \times \mathbb{Z}_{p}^{(n-3)}$.

Proof. Let $G$ ba an arbitrary non-abelian $p$-group of order $p^{n}$, we proceed by induction on $m$. The case $m=1$ follows from Lemma 3.8.

Therefore, we may assume that $m \geq 2$. Let $B$ be a central subgroup of order $p$ in $G$, we have

$$
\left|\mathcal{M}^{(2)}(G)\right|\left|B \cap \gamma_{3}(G)\right| \leq\left|\mathcal{M}^{(2)}(G / B)\right|\left|\left(B \otimes G^{a b}\right) \otimes G^{a b}\right|,
$$

by invoking Theorem 2.1(a). Using induction hypothesis

$$
\left|\mathcal{M}^{(2)}(G / B)\right| \leq p^{\frac{1}{3}(n-m)((n+2 m-5)(n-m-1)+3(m-2))},
$$


and so

$$
\begin{aligned}
\left|\mathcal{M}^{(2)}(G)\right| & \leq p^{\frac{1}{3}(n-m)((n+2 m-5)(n-m-1)+3(m-2))+3} p^{(n-m)^{2}} \\
& =p^{\frac{1}{3}(n-m)((n+2 m-2)(n-m-1)+3(m-1))+3}
\end{aligned}
$$

which completes the proof.

The following corollary is similar to that of $[\mathbf{2}, \mathbf{2 2}]$ for the order of the Schur multiplier.

COROLlary 3.10. Let $G$ be a p-group of order $p^{n}$. Then

(i) $\left|\mathcal{M}^{(2)}(G)\right|=p^{\frac{1}{3} n(n-1)(n+1)}$ if and only if $G$ is an elementary abelian $p$-group.

(ii) $\left|\mathcal{M}^{(2)}(G)\right|=p^{\frac{1}{3} n(n-1)(n-2)+3}$ if and only if $G \cong E_{1} \times \mathbb{Z}_{p}^{(n-3)}$. (iii) $\left|\mathcal{M}^{(2)}(G)\right| \leq p^{\frac{1}{3} n(n-1)(n-2)+2}$ provided that $G$ is neither elementary abelian nor
isomorphic to $E_{1} \times \mathbb{Z}_{p}^{(n-3)}$.

4. 2-Capability of extra-special $p$-groups. Capability of extra-special $p$-groups was first studied by Beyl et al. in [6], who determined all extra-special 1-capable groups. As mentioned the only capable groups in extra-special $p$-groups are $D_{8}$ and $E_{1}$. Here we will prove that the only 2 -capable groups in extra-special $p$-groups are these two ones too. That is, in extra special p-groups the notions "capable" and " 2 capable" are equivalent. Let $G$ be any 2-capable group, that is there exists a group $E$ with $G \cong E / Z_{2}(E)$ so we have $G \cong \frac{E / Z(E)}{Z(E / Z(E))}$, which shows $G$ is capable. Therefore all non-capable extra-special $p$-groups are not 2-capable as well. To prove the 2-capability of $D_{8}$ and $E_{1}$ we need the following proposition, which can be found in [6, Lemma 2.1(iv) and Proposition 1.2 ]. For the statement of this fact, we need some terminology from [6] as below.

Let $F / R$ be a free presentation for $G$ and $\pi: F /[R, F, F] \rightarrow G$ be the canonical surjection. The 2-central subgroup $Z_{2}^{*}(G)$ of $G$ is the image in $G$ of the second term of the upper central series of $F /[R, F, F]$. More precisely it is equal to $\pi\left(Z_{2}(F /[R, F, F])\right)$.

Proposition 4.1.

(i) A group $G$ is 2-capable if and only if $Z_{2}^{*}(G)$ is trivial,

(ii) If $N$ is a normal subgroup of $G$ contained in $Z_{2}^{*}(G)$, then the canonical

$$
\mathcal{M}^{(2)}(G) \hookrightarrow \mathcal{M}^{(2)}(G / N)
$$

homomorphisms is injection.

Beyls results can be extended to 2-capability as follows.

THEOREM 4.2. An extra-special p-group is 2-capable if and only if $G$ is isomorphic to either $D_{8}$ or $E_{1}$.

Proof. Let $G$ be the extra-special $p$-group of order $p^{3}$ and exponent $p$. We will show there is no nontrivial normal subgroup of $G$ for which the natural homomorphism $\mathcal{M}^{(2)}(G) \longrightarrow \mathcal{M}^{(2)}(G / N)$ is injective. Let $N$ be a nontrivial normal subgroup of $G$, so $G / N$ is an abelian $p$-group of order at most $p^{2}$ and hence, Theorem 3.7 shows $\left|\mathcal{M}^{(2)}(G / N)\right| \leq p^{2}$. Since $\left|\mathcal{M}^{(2)}(G)\right|=p^{5}$ by Theorem 3.6, $\mathcal{M}^{(2)}(G) \longrightarrow \mathcal{M}^{(2)}(G / N)$ fails 
to be injective and the result holds. The proof for the case $G=D_{8}$ is completely similar except that $\left|\mathcal{M}^{(2)}\left(D_{8}\right)\right|=2^{3}$.

\section{REFERENCES}

1. R. Baer, Representations of groups as quotient groups, I, II, and III, Trans. Am. Math. Soc. 58 (1945), 295-419.

2. Ya. G. Berkovich, On the order of the commutator subgroups and the Schur multiplier of a finite $p$-group, J. Algebra 144 (1991), 269-272.

3. F. R. Beyl, U. Felgner and P. Schmid, On groups occurring as center factor groups, $J$. Algebra 61 (1979), 161-177.

4. R. Brown, D. L. Johnson and E. F. Robertson, Some computations of non-abelian tensor products of groups, J. Algebra 111 (1987), 177-202.

5. R. Brown and J.-L. Loday, Van Kampen theorems for diagrams of spaces, Topology 26 (1987), 311-335. 405-428.

6. J. Burns and G. Ellis, On the nilpotent multipliers of a group, Math. Z. 226 (1997),

7. J. Burns and G. Ellis, Inequalities for Baer invariants of finite groups, Can. Math. Bull. 41(4) (1998), 385-391. $243-258$

8. G. Ellis, Tensor products and $q$-crossed modules, J. London Math. Soc. 51(2) (1995), 4177.

9. G. Ellis, On the Schur multiplier of p-groups, Commun. Algebra 9 (1999), 4173-

10. G. Ellis and J. Wiegold, A bound on the Schur multiplier of a prime power group, Bull. Aust. Math. Soc. 60 (1999), 191-196.

11. P. Hall, The classification of prime-power groups, J. Reine Angew. Math. 182 (1940), $130-141$. 157.

12. P. Hall, Verbal and marginal subgroups, J. Reine Angew. Math. 182 (1940), 156-

13. M. R. Jones, Multiplicators of p-groups, Math. Z. 127 (1972), 165-166.

14. M. R. Jones, Some inequalities for the multiplicator of a finite group, Proc. Am. Math. Soc. 39 (1973), 450-456.

15. G. Karpilovsky, The Schur multiplier, London Math. Soc. Monographs, New Ser., Vol. 2 (Clarendon Press, Oxford, 1987). 309-312.

16. A. S.-T. Lue, The Ganea map for nilpotent groups, J. London Math. Soc. 14 (1976),

17. B. Mashayekhy and M. R. R. Moghaddam, Higher Schur multiplicator of a finite abelian group, Algebra Colloq. 4(3) (1997), 317-322.

18. B. Mashayekhy and M. A. Sanati, On the order of nilpotent multipliers of finite pgroups, Commun. Algebra 33(7) (2005), 2079-2087.

19. M. R. R. Moghaddam, Some inequalities for the Baer invariant of a finite group, Bull. Iran. Math. Soc. 9 (1981), 5-10.

20. M. R. R. Moghaddam, On the Schur-Baer property, J. Aust. Math. Soc. Ser. A 31 (1981), 343-361.

21. M. R. R. Moghaddam, The Baer invariant of a direct product, Arch. Math. 33 (1980), 504-511.

22. P. Niroomand, On the order of Schur multiplier of non-abelian $p$-groups, J. Algebra 322 (2009), 4479-4482.

23. P. Niroomand, The Schur multiplier of $p$-groups with large derived subgroup, Arch. Math. 95 (2010), 101-103.

24. X. Zhou, On the order of Schur multipliers of finite p-groups, Commun. Algebra 1 (1994), 1-8. 